\title{
Generalized SyNCHRonization OF Nonlinear OSCILlATORS VIA OPCL COUPLING
}

\author{
Amit Mondal ${ }^{1}$ and Nurul Islam ${ }^{2}$ \\ ${ }^{1}$ Department of Mathematics, Jafarpur Kashinath High School, \\ P.O.- Champahati, P.S.- Sonarpur, Dist-24 Pgs(S), Pin - 743330 \\ West Bengal, India. \\ ${ }^{2}$ Department of Mathematics, Ramakrishna Mission Residential \\ College(Autonomous), \\ Narendrapur, Kolkata- 700103,West Bengal, India.
}

\begin{abstract}
In this communication, open-plus-closed-loop (OPCL) coupling method is applied to make generalized synchronization between two non-linear chaotic dynamical systems. For this reason, a transformation matrix is considered which can be chosen arbitrarily. We have used five different cases to establish our claim. Chaotic behaviours and the efficiency of the generalized synchronization using OPCL method are verified by numerical simulations.
\end{abstract}

\section{KEYWORDS}

Generalized Synchronization, OPCL coupling, Sprott System, Shimizu-Morioka System, Rossler System, Rikitake System

\section{INTRODUCTION}

Currently, there are many well-known control methods to stabilize non-linear chaotic dynamical systems. Out of all those control methods ([1]-[6],[9]), open-plus-closed-loop control method [11] is the most efficient method to make generalized synchronization (GS) for a coupled dynamical systems. This proposed method is insensitive with respect to system parameters which is one of the advantages of this method. This is a combination of open-loop system and closed-loop system. Open-loop means feed forward and closed-loop means feed backward. This combination gives us more flexibility to control and stabilize the dynamical systems. Using this method, the error term which is the difference between actual output and required output, reduces automatically by adjusting the system inputs. In this method, we are dealing with two systems known as master (drive) system and slave (response) system. Let $x=\left(x_{1}, x_{2}, x_{3}, \ldots, x_{n}\right)^{T} \in \mathrm{R}^{n}$ be the state variable of the master system and $y=\left(y_{1}, y_{2}, y_{3}, \ldots, y_{n}\right)^{T} \in \mathrm{R}^{n}$ be the state variable of the slave system. There exists another state known as goal state. Our aim is to reduce the difference between the goal system and slave system. Goal system depends on the master system such that $\sigma=K x$, where $\sigma=\left(\sigma_{1}, \sigma_{2}, \sigma_{3}, \ldots, \sigma_{n}\right)^{T} \in \mathrm{R}^{n}$, the state variable of the goal system and $\mathrm{K}$ is a transformation matrix of order $\mathrm{n}$, chosen arbitrarily. Here, in this communication, we will choose five different forms of the matrix $\mathrm{K}$. In section 3, we will study five different cases corresponding to different forms of the matrix K. In the first case, the elements of K are taken constants. In case-II, periodic functions are considered as the elements of K-matrix. The state 
variables of the master system will be taken as the elements of K-matrix in case-III. For the next case, the elements of K-matrix are taken as the state variables of the other dynamical system. Finally, in case- $\mathrm{V}$, discussion is made where one dynamical system drives another dynamical system which is totally different in nature with the former dynamical system along with the Kmatrix whose elements are the state variables of the other dynamical system. This last case is the most interesting part of this paper. Open-plus-closed-loop coupling method is very useful in engineering science, chemical reactions, quantum physics, lasers, electronic circuits, secure communication, microwave oscillators, electrical clothes drier etc.

\section{DESCRIPTION OF OPCL CONTROLLER FOR GS}

To describe this method, let us take a non-linear dynamical system as the master system given below:

$$
\dot{x}=\phi(x)
$$

where $\mathrm{x}\left(\in \mathrm{R}^{n}\right)$ is the state variable of the master system \& $\phi: \mathrm{R}^{n} \rightarrow \mathrm{R}^{n}$.

Next, we consider the slave system given by the following dynamics

$$
\dot{y}=\psi(y)+u
$$

where $\mathrm{y}\left(\in \mathrm{R}^{n}\right)$ is the state variable of the slave system \& $\psi: \mathrm{R}^{n} \rightarrow \mathrm{R}^{n}$ and $\mathrm{u}$ is the control input.

Let the generalized synchronization error be defined as

$$
\begin{gathered}
e=y-K x \\
\text { or, } y=\sigma+e, \text { where } \sigma=K x
\end{gathered}
$$

Now, using Taylor's expansion of a function, we have from equation (2) \& (3)

$$
\begin{aligned}
\dot{y} & =\psi(\sigma+e)+u \\
& =\psi(\sigma)+\frac{\partial \psi(\sigma)}{\partial \sigma} e+u
\end{aligned}
$$

neglecting second and higher orders of e to be very small.

$$
\therefore \dot{y}=\psi(\sigma)+J(\sigma) e+u \text {, }
$$

where $J(\sigma)$ is the Jacobian of the flow $\psi(\sigma)$.

Let us define,

$$
u=\dot{\sigma}-\psi(\sigma)+\mu e, \text { where } \mu=V-J(\sigma)
$$

and $\mathrm{V}$ is a matrix of order $\mathrm{n}$. 
Using (4) and (5), one gets easily

$$
\dot{e}=V e
$$

which gives the error dynamics.

Now, the error dynamics(6) is globally asymptotically stable if V-matrix is Hurwitz. Hence, we can conclude that generalized synchronization between the system (1) and (2) does not depend on $\mathrm{K}$-matrix, it depends on the V-matrix. In this communication, we are choosing the elements of the V-matrix are similar to the elements of the Jacobian matrix of the slave system except all those elements which carry the state variables of the slave system. In this situation, we take constant value $w_{i}(i=1,2,3, \ldots)$ instead of the state variable of the slave system for which $\mathrm{V}$-matrix is Hurwitz, i.e., all the eigen values of $\mathrm{V}$ have negative real parts. Accordingly the error dynamics (6) is globally asymptotically stable. Finally, we claim that the generalized synchronization of the master-slave system is made successfully.

\section{EXAMPLES OF GS USING OPCL CONTROLLER}

Case-I : According to previous section, we first consider a non-linear chaotic Sprott system L [7] as the master system given by

$$
\dot{x}=\phi(x), x \in \mathrm{R}^{3}
$$

where

$$
\phi(x)=\left(\begin{array}{c}
x_{2}+a_{1} x_{3} \\
b_{1} x_{1}^{2}-x_{2} \\
c_{1}-x_{1}
\end{array}\right), a_{1}, b_{1} \& c_{1} \text { are the parameters }
$$

The slave system is taken as the mismatch Sprott system L

$$
\dot{y}=\psi(y)+u, \quad y \in \mathrm{R}^{3}
$$

where

$$
\psi(y)=\left(\begin{array}{c}
y_{2}+a_{2} y_{3} \\
b_{2} y_{1}^{2}-y_{2} \\
c_{2}-y_{1}
\end{array}\right), a_{2}, b_{2} \& c_{2} \text { are the parameters }
$$

and $u=\left(u_{1}, u_{2}, u_{3}\right)^{t} \in \mathrm{R}^{3}$ is the controller. 
Let

$$
K=\left(\begin{array}{ccc}
1 & -0.8 & -2.7 \\
0 & 0.5 & -2.3 \\
0.5 & 0 & 0.7
\end{array}\right)
$$

Using $\sigma=K x$, we have the goal dynamics as

$$
\left.\begin{array}{rl}
\dot{\sigma}_{1} & =\dot{x}_{1}-0.8 \dot{x}_{2}-2.7 \dot{x}_{3} \\
\dot{\sigma}_{2} & =0.5 \dot{x}_{2}-2.3 \dot{x}_{3} \\
\dot{\sigma}_{3} & =0.5 \dot{x}_{1}+0.7 \dot{x}_{3}
\end{array}\right\}
$$

The Jacobian matrix of the slave system is given by

$$
J(y)=\left(\begin{array}{ccc}
0 & 1 & a_{2} \\
2 b_{2} y_{1} & -1 & 0 \\
-1 & 0 & 0
\end{array}\right)
$$

Then, $\mathrm{V}$ can be taken as,

$$
V=\left(\begin{array}{ccc}
0 & 1 & a_{2} \\
2 b_{2} w_{1} & -1 & 0 \\
-1 & 0 & 0
\end{array}\right) \text {, where } w_{1} \text { is chosen arbitrarily }
$$

so that V is Hurwitz.

Hence, from equation (6), the error dynamics becomes

$$
\left.\begin{array}{l}
\dot{e}_{1}=e_{2}+a_{2} e_{3} \\
\dot{e}_{2}=2 b_{2} w_{1} e_{1}-e_{2} \\
\dot{e}_{3}=-e_{1}
\end{array}\right\}
$$

Then, one can easily obtain,

$$
\mu=\left(\begin{array}{ccc}
0 & 0 & 0 \\
2 b_{2}\left(w_{1}-\sigma_{1}\right) & 0 & 0 \\
0 & 0 & 0
\end{array}\right), \text { where } w_{1} \text { is chosen arbitrarily }
$$

so that V is Hurwitz. 
International Journal on Cybernetics \& Informatics (IJCI) Vol. 3, No. 2, April 2014

Now, using (5), the control input is found as

$$
\left.\begin{array}{l}
u_{1}=\dot{\sigma}_{1}-\sigma_{2}+a_{2} \sigma_{3} \\
u_{2}=\dot{\sigma}_{2}-b_{2} \sigma_{1}^{2}+\sigma_{2}+2 b_{2}\left(w_{1}-\sigma_{1}\right) e_{1} \\
u_{3}=\dot{\sigma}_{3}-c_{2}+\sigma_{1}
\end{array}\right\}
$$
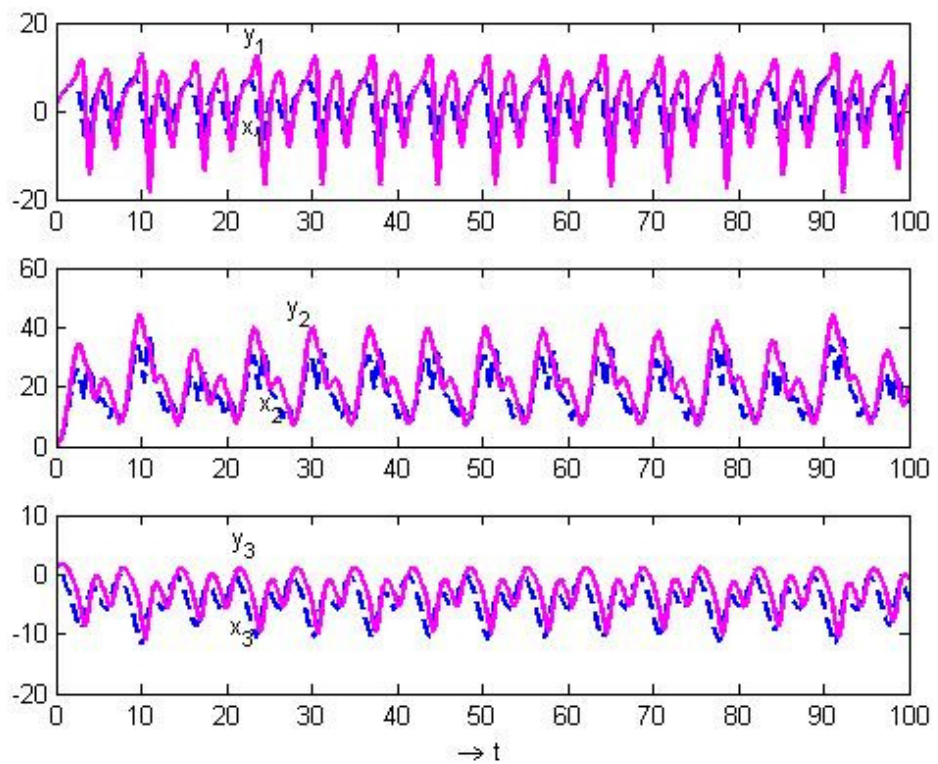

Figure 1: case-I: master system (x) \& slave system (y) with respect to time
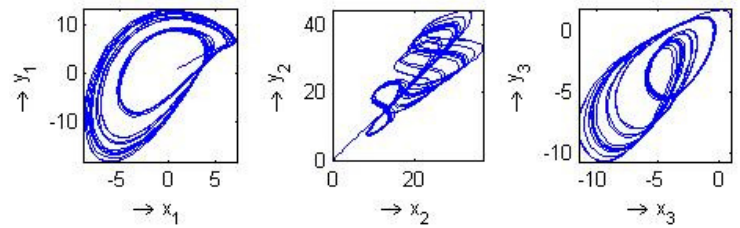

(a)
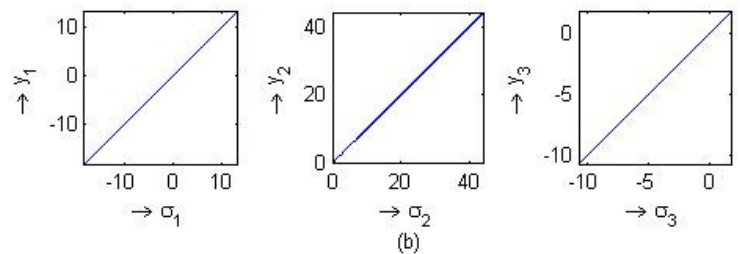

Figure 2: case-I: (a) x vs y plot (b) $\sigma$ vs y plot 
Case-II : Here, the master system and the slave system are taken to be same as the system (7) \& (8) respectively.

In this case, $\mathrm{K}$ is taken to be a $3 \times 3$ matrix with periodic function as its elements given below :

$$
K=\left(\begin{array}{ccc}
0 & -0.2 \cos (0.6 t) & 3.5 \\
1 & 1 & 0 \\
-\sin (0.1 t) & -0.5 & 0
\end{array}\right)
$$

Thus, we have the goal dynamics as $\dot{\sigma}=K \dot{x}+\dot{K} x$, which yields

$$
\left.\begin{array}{l}
\dot{\sigma}_{1}=-0.2 \cos (0.6 t) \dot{x}_{2}-3.5 \dot{x}_{3}-0.12 \sin (0.6 t) x_{2} \\
\dot{\sigma}_{2}=\dot{x}_{1}+\dot{x}_{2} \\
\dot{\sigma}_{3}=-\sin (0.1 t) \dot{x}_{1}-0.5 \dot{x}_{2}-0.1 \cos (0.1 t) x_{1}
\end{array}\right\}
$$

The error dynamics is same as the previous case, because the Jacobian matrix of the slave system remains the same.

Hence, using(5), the control input $u$ is given by

$$
\left.\begin{array}{l}
u_{1}=\dot{\sigma}_{1}-\sigma_{2}+a_{2} \sigma_{3} \\
u_{2}=\dot{\sigma}_{2}-b_{2} \sigma_{1}^{2}+\sigma_{2}+2 b_{2}\left(w_{1}-\sigma_{1}\right) e_{1} \\
u_{3}=\dot{\sigma}_{3}-c_{2}+\sigma_{1}
\end{array}\right\}
$$
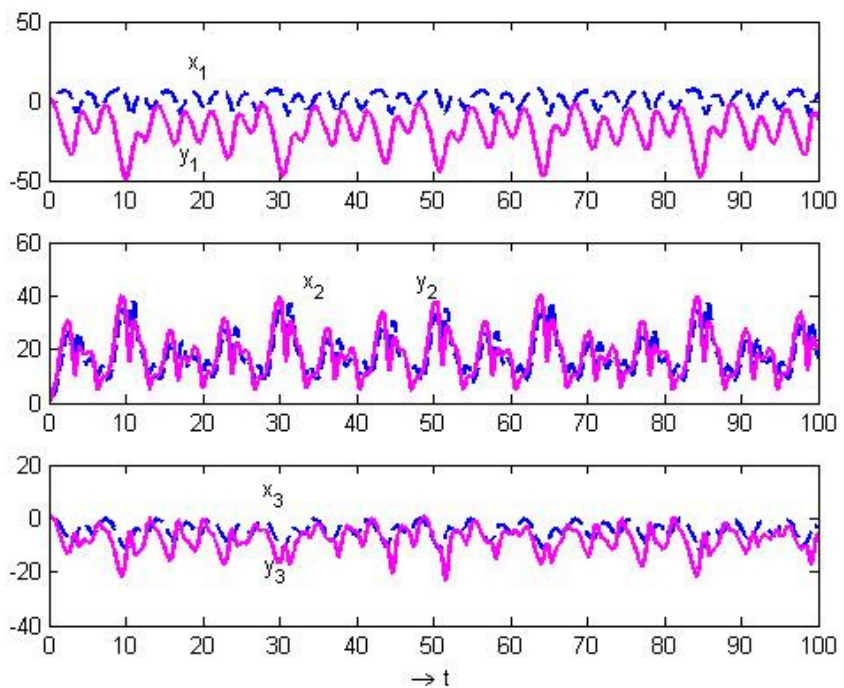

Figure 3: case-II: master system (x) \& slave system (y) with respect to time 

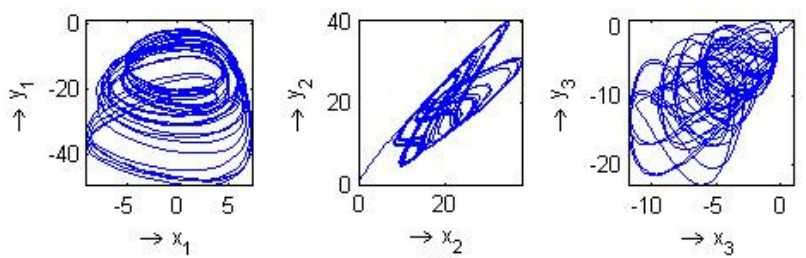

(a)
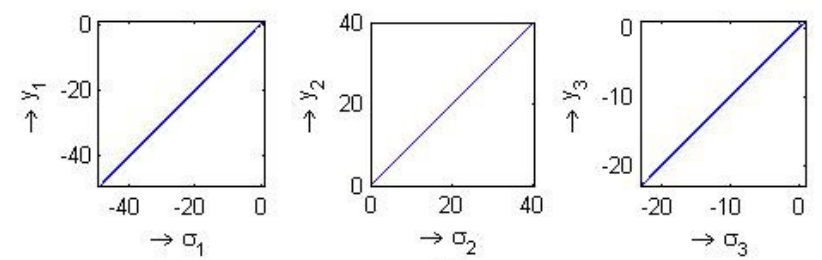

(b)

Figure 4: case-II: (a) x vs y plot (b) $\sigma$ vs y plot

Case- III : Let $\mathrm{K}$ be a $3 \times 3$ matrix containing the state variables of the master system (7) as its elements and the slave system remains unchanged,

where

$$
K=\left(\begin{array}{ccc}
1 & 0.03 x_{1} & 0 \\
-0.02 x_{1} & -0.1 x_{3} & 0 \\
0 & 0.01 x_{2} & 0
\end{array}\right)
$$

Then, the system of goal dynamics is obtained as

$$
\left.\begin{array}{l}
\dot{\sigma}_{1}=\dot{x}_{1}+0.03\left(\dot{x}_{1} x_{2}+x_{1} \dot{x}_{2}\right) \\
\dot{\sigma}_{2}=-0.04 x_{1} \dot{x}_{1}-0.1\left(\dot{x}_{3} x_{2}+x_{3} \dot{x}_{2}\right) \\
\dot{\sigma}_{3}=0.02 \dot{x}_{2} x_{2}
\end{array}\right\}
$$

Here, the error dynamics remains similar as the previous case and the controller $u$ as follows :

$$
\left.\begin{array}{l}
u_{1}=\dot{\sigma}_{1}-\sigma_{2}+a_{2} \sigma_{3} \\
u_{2}=\dot{\sigma}_{2}-b_{2} \sigma_{1}^{2}+\sigma_{2}+2 b_{2}\left(w_{1}-\sigma_{1}\right) e_{1} \\
u_{3}=\dot{\sigma}_{3}-c_{2}+\sigma_{1}
\end{array}\right\}
$$



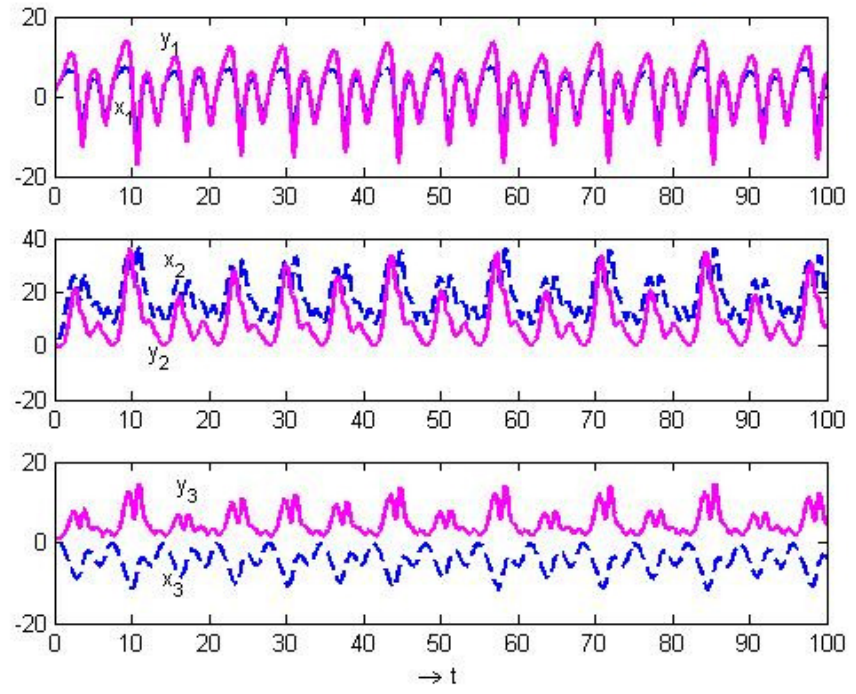

Figure 5: case-III: master system (x) \& slave system (y) with respect to time
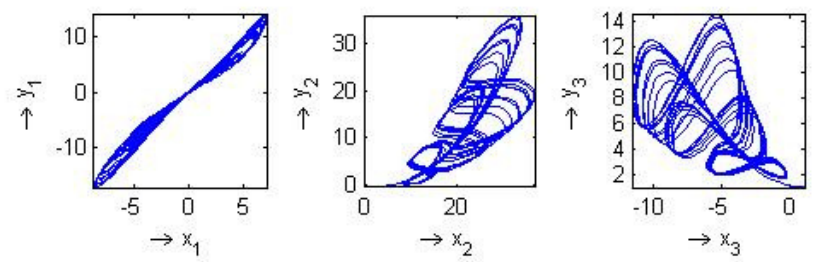

(a)
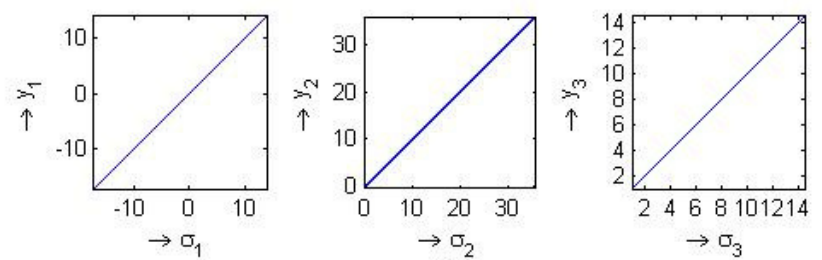

(b)

Figure 6: case-III: (a) x vs y plot (b) $\sigma$ vs y plot

Case- IV : In this case, the elements of K-matrix are chosen so that it contains the state variables of Shimizu-Morioka system whereas the master-slave system are taken to be the mismatched coupled Sprott system L given by the system (7) \& (8).

The Shimizu-Morioka system [10] is given by

$$
\left.\begin{array}{ll}
\dot{z}_{1} & =\delta z_{2} \\
\dot{z}_{2} & =\delta\left(z_{1}-\lambda z_{2}-z_{1} z_{3}\right) \\
\dot{z}_{3} & =\delta\left(-\rho z_{3}+z_{1}^{2}\right)
\end{array}\right\}
$$


where $\lambda \& \rho$ are the positive parameters and $\delta=0.02$ for which the original system of equation is slightly being changed without loss of generality.

Let

$$
K=\left(\begin{array}{ccc}
-3.5 z_{2} & 0 & 0 \\
0 & 0.1 z_{1} & 3 \\
-1 & 0 & 2.7 z_{3}
\end{array}\right)
$$

Then, the goal dynamics is found as

$$
\left.\begin{array}{l}
\dot{\sigma}_{1}=-3.5\left(\dot{x}_{1} z_{2}+x_{1} \dot{z}_{2}\right) \\
\dot{\sigma}_{2}=3 \dot{x}_{3}+0.1\left(\dot{z}_{1} x_{2}+z_{1} \dot{x}_{2}\right) \\
\dot{\sigma}_{3}=-\dot{x}_{1}+2.7\left(\dot{x}_{3} z_{3}+x_{3} \dot{z}_{3}\right)
\end{array}\right\}
$$

Using (17) and (5), we get the controller $\mathrm{u}$ as,

$$
\left.\begin{array}{l}
u_{1}=\dot{\sigma}_{1}-\sigma_{2}+a_{2} \sigma_{3} \\
u_{2}=\dot{\sigma}_{2}-b_{2} \sigma_{1}^{2}+\sigma_{2}+2 b_{2}\left(w_{1}-\sigma_{1}\right) e_{1} \\
u_{3}=\dot{\sigma}_{3}-c_{2}+\sigma_{1}
\end{array}\right\}
$$

where $e=\left(e_{1}, e_{2}, e_{3}\right)^{t} \in \mathrm{R}^{3}$, the state variables of the error system (10).
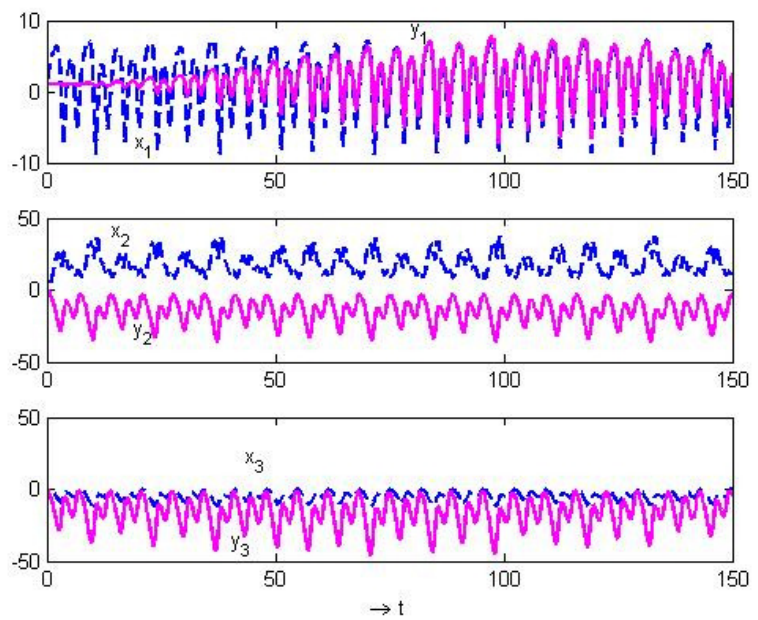

Figure 7: case-IV: master system (x) \& slave system (y) with respect to time 

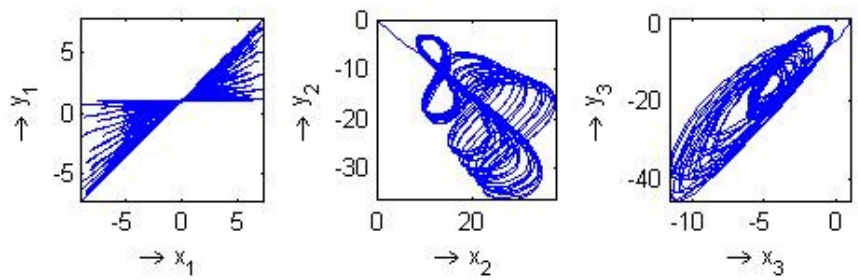

(a)
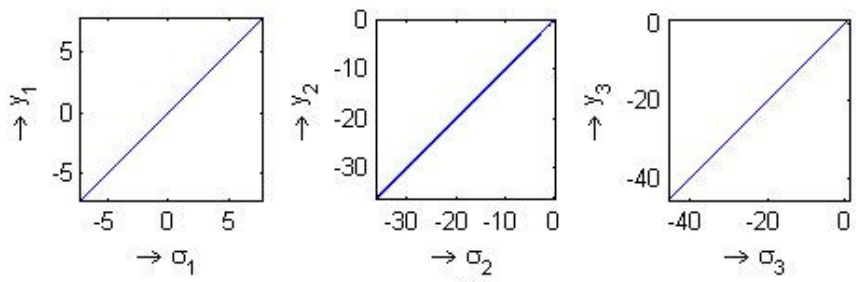

(b)

Figure 8: case-IV: (a) x vs y plot (b) $\sigma$ vs y plot

Case- V : Here, Rikitake system drives Sprott L system (8) with transformation matrix K consisting state variables of Rossler system.

The non-linear Rikitake system [8] considered as the master system given by

$$
\left.\begin{array}{ll}
\dot{r}_{1} & =\alpha r_{1}+r_{2} r_{3} \\
\dot{r}_{2} & =-\alpha r_{2}+\left(r_{3}-\beta\right) r_{1} \\
\dot{r}_{3} & =1-r_{1} r_{2}
\end{array}\right\} \text {, where } \alpha, \beta \text { are the parameters. }
$$

To construct the K-matrix, we consider the Rossler dynamical system [9] as

$$
\left.\begin{array}{l}
\dot{s}_{1}=-s_{2}-s_{3} \\
\dot{s}_{2}=s_{1}+l s_{2} \\
\dot{s}_{3}=m+s_{3}\left(s_{1}-p\right)
\end{array}\right\} \text {, where } l, m, p \text { are the parameters. }
$$

Let us take,

$$
K=\left(\begin{array}{ccc}
0.75 s_{1} & 0.45 s_{3} & -0.01 s_{1} \\
0 & -0.35 s_{1} & -0.1 s_{1} \\
-0.01 s_{3} & 0 & 0.02 s_{2}
\end{array}\right)
$$

In this case, the goal variable can be selected as $\sigma=K r, r=\left(r_{1}, r_{2}, r_{3}\right)^{t} \in \mathrm{R}^{3}$, the state variable of the master system (19). 
Then, the goal dynamics is given by

$$
\left.\begin{array}{l}
\dot{\sigma}_{1}=0.75\left(s_{1} \dot{r}_{1}+\dot{s}_{1} r_{1}\right)+0.45\left(s_{3} \dot{r}_{2}+\dot{s}_{3} r_{2}\right)-0.01\left(s_{1} \dot{r}_{3}+\dot{s}_{1} r_{3}\right) \\
\dot{\sigma}_{2}=-0.35\left(s_{1} \dot{r}_{2}+\dot{s}_{1} r_{2}\right)-0.1\left(s_{1} \dot{r}_{3}+\dot{s}_{1} r_{3}\right) \\
\dot{\sigma}_{3}=-0.01\left(s_{3} \dot{r}_{1}+\dot{s}_{3} r_{1}\right)+0.02\left(s_{2} \dot{r}_{3}+\dot{s}_{2} r_{3}\right)
\end{array}\right\}
$$

Hence, $\mathrm{u}$, the control input of the slave system (Sprott L system) is calculated as

$$
\left.\begin{array}{l}
u_{1}=\dot{\sigma}_{1}-\sigma_{2}+a_{2} \sigma_{3} \\
u_{2}=\dot{\sigma}_{2}-b_{2} \sigma_{1}^{2}+\sigma_{2}+2 b_{2}\left(w_{1}-\sigma_{1}\right) e_{1} \\
u_{3}=\dot{\sigma}_{3}-c_{2}+\sigma_{1}
\end{array}\right\}
$$

where $e=\left(e_{1}, e_{2}, e_{3}\right)^{t} \in \mathrm{R}^{3}$, the state variables of the error system which remains same with the previous four cases because $\mathrm{V}$-matrix remains unchanged.
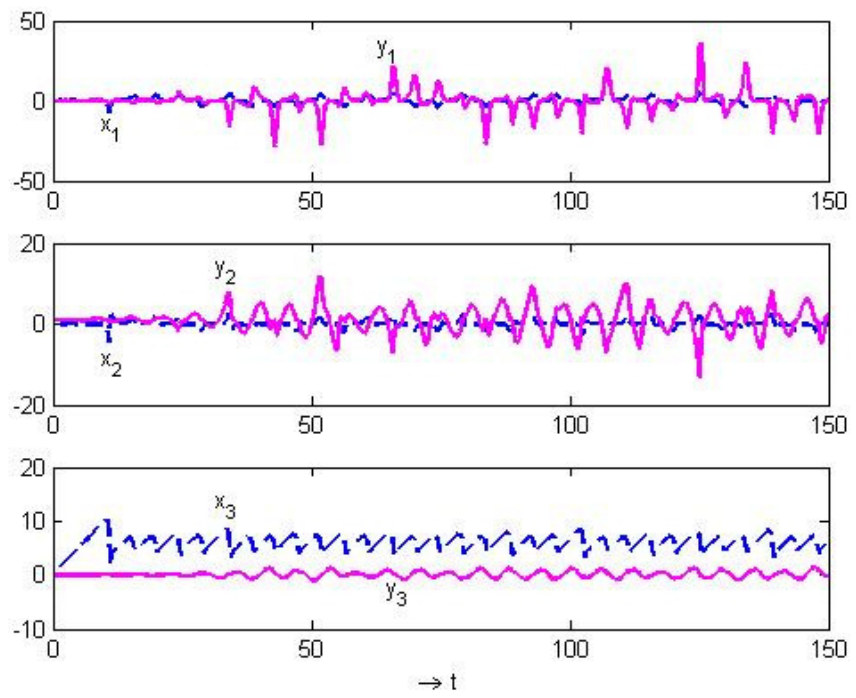

Figure 9: case-V: master system (x) \& slave system (y) with respect to time
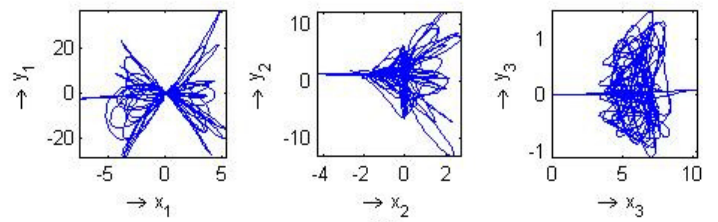

(a)
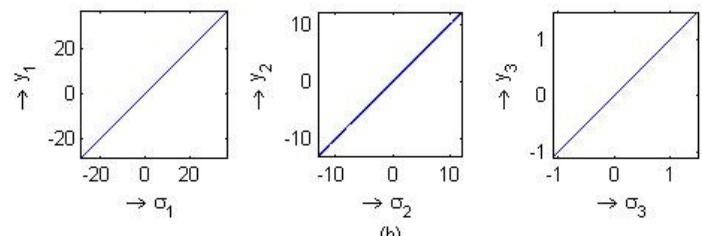

Figure 10: case-V: (a) x vs y plot (b) $\sigma$ vs y plot 
International Journal on Cybernetics \& Informatics (IJCI) Vol. 3, No. 2, April 2014

\section{NuMERICAL RESUltS \& DisCUSSIONS}

Here, we will discuss the previous section numerically with the help of matlab software.

Sprott found the chaotic nature for the master system (7) when $a_{1}=3.9, b_{1}=0.9, c_{1}=1$.

To make non-identical coupled Sprott $\mathrm{L}$ system for the slave system(8), we take $a_{2}=4.4, b_{2}=1.6, c_{2}=1.7$.

V-matrix reduces to Hurwitz if we take $w_{1}=-4.5$.

In Shimizu-Morioka system(16), we consider $\lambda=0.799 \& \rho=0.54$ for showing its chaotic nature.

In the non-linear Rikitake system(19), there exists two parameters $\alpha \& \beta$. Let $\alpha=2, \beta=5$.

Finally, we choose $l=m=0.2 \& p=5.7$ for the Rossler system given by equation(20).

Figures fig.1, fig.3, fig.5, fig.7 and fig.9 represents the graph of the master and the slave system with respect to time respectively.

In figures fig.2(a), fig.4(a), fig.6(a), fig.8(a) and fig.10(a), we have plotted $x_{i}$ vs $y_{i}, i=1,2,3$ for all the cases I through $\mathrm{V}$.

From the relation (3), we can claim that the error term goes to zero after some finite time by reducing the difference between goal variables and slave variables. To establish our claim, we have drawn fig.2(b), fig.4(b), fig.6(b), fig.8(b) and fig.10(b).

\section{CONCLUSIONS}

In this paper, we have successfully established the generalized synchronization between the master (drive) system \& the slave (response) system via OPCL method. This method is mostly independent of the system parameters. This method has so many applications in practical life, for example, microwave oscillators, electrical cloths drier etc. In engineering sciences, it is also very useful.

\section{REFERENCES}

[1] J H Park (2005), "Controlling chaotic systes via nonlinear feedback control”, Chaos,Solitons and Fractals, Vol.23, No.3, pp1049-1054.

[2] I D Landau, R Lozano, M M'Saad and A Karimi (2011), Adaptive control, communication and control engineering, Springer-Verlag London Limited.

[3] S Pal (2012), "Synchronization of coupled hyper-chaotic systems", Differential Geometry and Dynamical Systems (DGDS), Vol.14, pp117-124.

[4] C Liqun and L Yanzhu (1998), "Control of Lorenz chaos by the exact linearization", Applied Mathematics and Mechanics, Vol.19, No.1, pp67-73.

[5] S Vaidyanathan (2013), "Sliding Mode Controller design for the anti-synchronization of hyperchaotic Lu systems”, International Journal on Cybernetics and Informatics (IJCI), Vol.2, No.1, pp31-38.

[6] E M Shahverdiev and K A Shore (2009), "Impact of modulated multiple optical feedback time delays on laser diode chaos synchronization”, Optics communication,Vol. 282, pp3568-3572. 
[7] A Mondal and N Islam (2013), "Chaos synchronization of coupled Sprott model L", International Journal of Pure and Applied Science and Technology(IJPAST), Vol.16, No.2, pp32-38.

[8] M Javidi and N Nyamoradi (2013), "Numerical Chaotic Behavior of the Fractional Rikitake System", World Journal of Modelling and Simulation, Vol.9, No.2, pp120-129.

[9] H.N. Agiza and M.T. Yassen (2001), "Synchronization of Rossler and Chen chaotical dynamical systems using active control”, Phys. Lett. A, Vol.278, No.4, pp191-197.

[10] N Islam, B Islam and H P Mazumdar (2011), "Generalized chaos synchronization of unidirectionally coupled Shimizu-Morioka dynamical systems",Differential Geometry and Dynamical System (DGDS), Vol.13, pp101-106.

[11] P K Roy, C Hens, I Grosu and S K Dana (2011), "Engineering generalized synchronization in chaotic oscillators", Chaos 21, 013106

\section{Authors}

Amit Mondal received B.Sc(HONS.) degree in Mathematics from Asutosh College, Calcutta University, West Bengal, India. He also received the Master in Science in Pure Mathematics from Ballygunge Science College, Calcutta University, West Bengal, India. He is currently working in Jafarpur Kashinath High School, Champahati, Kolkata, West Bengal,India, as a Teacher in Mathematics. His main research interests include Non-linear dynamics, Stability,Various types of Control in Chaos and Synchronization.

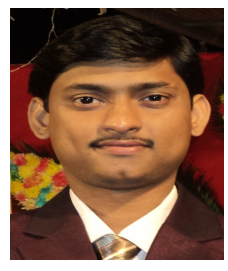

Nurul Islam received his M.Sc degree in applied mathematics from University of Calcutta (India,1979) and his Ph.D degree from Jadavpur University (India, 1996), in the field of fluid mechanics, specializing in turbulence under the doctoral supervision of Prof. H.P. Mazumdar, Indian Statistical Institute (Kolkata, India). He is presently pursuing the field of Chaos and non-linear dynamics, and is currently supervising 5 doctoral candidates in this area of research. $\mathrm{He}$ has also co-supervised Government funded projects (University Grants Commission, India) with Prof. S. Sarkar, Dept. of Electronics and Telecommunication- Jadavpur University, as a principal investigator, in investigating areas like, digital image processing and

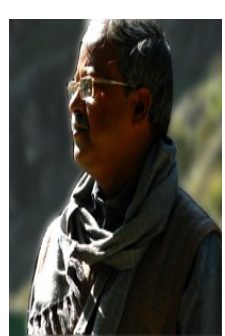
watermarking with applications in forensic investigations. Since 1982, he has been a faculty member in the Department of Mathematics, Ramakrishna Mission Residential College (autonomous), (Narendrapur, Kolkata, India), where he is currently an Associate professor and Head of the Department. He is also a member of the executive council of the prestigious Calcutta Mathematical Society, India. 